\title{
Myxoid Liposarcoma: Treatment Outcomes from Chemotherapy and Radiation Therapy
}

\author{
Varun Chowdhry iD, ${ }^{1}$ Saveli Goldberg, ${ }^{2}$ Thomas F. DeLaney, ${ }^{2}$ Gregory M. Cote, ${ }^{3}$ \\ Ivan Chebib, ${ }^{4}$ Jason Kim, ${ }^{5}$ Santiago A. Lozano-Calderón, ${ }^{5}$ \\ and Karen De Amorim Bernstein ${ }^{2}$
}

${ }^{1}$ Department of Radiation Medicine, Roswell Park Comprehensive Cancer Center, Buffalo, NY, USA

${ }^{2}$ Department of Radiation Oncology, Massachusetts General Hospital, Boston, MA 02114, USA

${ }^{3}$ Division of Hematology/Oncology, Massachusetts General Hospital, Boston, MA 02114, USA

${ }^{4}$ Department of Pathology, Massachusetts General Hospital, Boston, MA 02114, USA

${ }^{5}$ Department of Orthopedic Oncology, Massachusetts General Hospital, Boston, MA 02114, USA

Correspondence should be addressed to Varun Chowdhry; varun.chowdhry@roswellpark.org

Received 3 August 2018; Accepted 10 October 2018; Published 1 November 2018

Academic Editor: C. Verhoef

Copyright (c) 2018 Varun Chowdhry et al. This is an open access article distributed under the Creative Commons Attribution License, which permits unrestricted use, distribution, and reproduction in any medium, provided the original work is properly cited.

\begin{abstract}
Introduction. Myxoid liposarcoma (MLS) is a subtype of liposarcoma characterized morphologically by lipomatous differentiation with a myxoid stroma. The purpose of this study was to review clinical and pathological information for patients treated for MLS at our institution to better understand neoadjuvant and adjuvant therapy. Materials and Methods. An institutional database of sarcomas was queried for patients who were treated for MLS at our institution between 1992 and 2013. Survival curves were constructed using Kaplan-Meier analysis, and univariate and multivariate statistics were performed using the Cox-proportional hazards model and using linear regression. Results. A total of 85 patients with myxoid liposarcoma were identified. The mean and median histologic response rate to treatment for patients who received preoperative radiation therapy was $77.6 \%$. Five-year disease-free survival, distant metastasis-free survival, local recurrence-free survival, and overall survival were $78.6 \%(95 \% \mathrm{CI}$ : 67.8-86.1), 84.7\% (95\% CI: 74.5-91.0), 95.6\% (95\% CI: 86.9-98.6), and 87.5\% (95\% CI: 77.2-93.3) respectively. On univariate analysis, there was a trend towards higher necrosis or treatment response rates in patients who received concurrent chemotherapy, 84.7\% (95\% CI: 75.9-93.4) and 69.5\% (95\% CI: 55.1-83.8), $p=0.061$. Tumor size was associated with inferior disease-free and overall survival. Hazard ratio for disease-free survival is 1.08 (per $\mathrm{cm})(95 \%$ CI: 1.01-1.16), $p=0.019$. Conclusions. Myxoid liposarcoma exhibits histological response to chemotherapy and radiation therapy. Tumor size appears to be greatest predictor of long-term disease control and overall survival. We were not able to show that chemotherapy provides a clinical benefit with regard to local control, disease-free survival, or overall survival. However, it is important to note that the selected usage of chemotherapy in the highest risk patients confounds this analysis. Further investigation is needed to help better determine the optimal use of chemotherapy in this group of patients.
\end{abstract}

\section{Introduction}

Myxoid liposarcoma (MLS) is a subtype of liposarcoma that represents a distinct pathological entity characterized morphologically by tumor cells within a myxoid stroma with a rich, branching thin-walled vasculature, and focal lipomatous differentiation. The MLS subtype represents approximately $1 / 3$ of all liposarcomas and $10 \%$ of adult soft tissue sarcomas [1]. MLS is associated with chromosomal translocations consisting of the FUS and DDIT3 (CHOP) genes $\mathrm{t}(12 ; 16)(\mathrm{q} 13 ; \mathrm{p} 11)$ or the EWS and DDIT3 (CHOP) genes $t(12 ; 22)$ (q13; q12) [2]. There is evidence to suggest that MLS is both radioresponsive and radiosensitive [3]. Marked reduction of tumor volume has been noted during 
treatment, with one series showing a median reduction in tumor volume from the start to end of treatment of 59\% [4].

Comparative analysis of MLS versus other sarcoma subtypes suggests greater response rates in MLS with the addition of anthracycline-based chemotherapy [5]. In one series, there is a suggestion that doxorubicin and ifosfamide can result in favorable long-term outcomes [6]. However, there is relatively limited information on factors that predict for overall outcome in MLS, particularly with regard to the benefits of combined trimodality therapy. The purpose of this single institution MLS series is to evaluate clinical variables that may predict for improved outcomes and thereby help guide management or future clinical trials.

\section{Materials and Methods}

After IRB approval, our oncology registry database was queried for patients who were treated for MLS at our institution between 1992 and 2013. Only patients with no evidence of metastatic disease treated with definitive limbsalvage therapy were included in this series. All patients had pathological confirmation by a sarcoma-specialized pathologist either through secondary review of slides from another institution or through direct pathological sampling obtained at our institution. All patients were evaluated in a multidisciplinary care setting for indications and suitability to receive preoperative chemotherapy and radiation therapy. Patients treated with definitive therapy for their disease were included in this analysis. Sixty-nine out of 85 patients $(81 \%)$ had the initial biopsy performed at our institution, and seventy-nine patients (93\%) had definitive surgical resection performed at our institution. Patients diagnosed with MLS, including high-grade MLS (formerly myxoid/round cell liposarcoma), were included in this analysis. Demographic, clinical, radiographic, pathologic, and treatment outcomes were captured. Statistical analysis using both univariate and multivariate models were conducted using SAS software (SAS version 9.4; 100 SAS Campus Drive, Cary, NC 27513). Survival curves were constructed using Kaplan-Meier analysis, and univariate and multivariate statistics were performed using the Cox-proportional hazards model and using linear regression.

\section{Results}

Demographic information for patients in this series is listed in Table 1. Seventy-three of 85 patients had sarcomas of the lower extremity $(85.9 \%)$, with complete breakdown by location shown in Table 1 . The median follow-up for patients in this series was 85.2 months (range, 4-250 months). Sixty-seven (78.8\%) patients were treated with preoperative radiotherapy, and 15 (17.6\%) patients were treated with postoperative radiation therapy, generally due to close or positive postoperative margins. Ten patients $(11.8 \%)$ in this series did not receive any radiotherapy. Seven patients $(8.2 \%)$ were treated with both pre- and postoperative radiation therapy. Patients were treated with either $2 \mathrm{D}$ radiotherapy prior to $2000,3 \mathrm{D}$ conformal radiation therapy (3D-CRT), or intensity-
TABLe 1: Demographic information.

\begin{tabular}{ll}
\hline Total number of patients & 85 \\
Male & 48 \\
Female & 37
\end{tabular}

Median age (years)

Patients treated with preoperative radiation therapy

Patients treated with postoperative

radiation therapy

Patients treated with pre- and postoperative

radiation therapy

Patients treated with postoperative

radiation therapy only

Patients not treated with radiation therapy

Number of patients treated with

chemotherapy

42 (range, $18-88$ years)

$67(78.8 \%)$

$15(17.6 \%)$

$7(8.2 \%)$

$8(9.4 \%)$

$10(11.8 \%)$

39

Location of tumor

Right thigh

Left thigh

Right lower leg

Left lower leg

Right knee

Buttock

Left knee

Abdomen

Paraspinal

Chest wall

Left shoulder

Back

Vulva

Head and neck

Tumor size

Mean $(\mathrm{cm})$

Median $(\mathrm{cm})$

Range $(\mathrm{cm})$

Median preoperative radiation dose (cGy)

Median postoperative radiation dose (cGy)

Median postoperative dose in patients

receiving preoperative radiation therapy

(cGy)

Median postoperative dose in patients not receiving preoperative radiation therapy

(cGy)

Margin status (number of patients)

Gross positive

Microscopically positive

Close $(<1 \mathrm{~mm})$

Negative

Margin status not reported

Local failures

Local failures (patients treated with

preoperative radiation therapy)

Local failures (patients not treated with

preoperative therapy)

$30(35.3 \%)$

$28(32.9 \%)$

$6(7.1 \%)$

$4(4.7 \%)$

$3(3.5 \%)$

$3(3.5 \%)$

$2(2.4 \%)$

$2(2.4 \%)$

$1(1.2 \%)$

$1(1.2 \%)$

$1(1.2 \%)$

$1(1.2 \%)$

$1(1.2 \%)$

$1(1.2 \%)$

12.0

10.0

1.3-35

5000 (range,

2000-7100)

1600 (range,

1000-7020)

1600 (range,

1000-2200)

6150 (range,

5940-7020)

$2(2.4 \%)$

$12(14.1 \%)$

$50(58.8 \%)$

$19(22.3 \%)$

$2(2.4 \%)$

$4 / 85(4.7 \%)$

2/67 (3.0\%)

$2 / 18(11 \%)$

modulated radiotherapy (IMRT). Thirty-nine (45.9\%) patients were treated with preoperative chemotherapy, and $22(25.9 \%)$ of these patients received at least one cycle of postoperative chemotherapy. The chemotherapy regimens were almost entirely anthracycline-based, including 
mesna, doxorubicin, ifosfamide, and dacarbazine (MAID) or doxorubicin, ifosfamide, and mesna (AIM) chemotherapy, with the exception of one patient who received bevacizumab alone on a clinical trial.

Five-year disease-free survival, distant metastasis-free survival, local control, and overall survival were $78.6 \%$ (95\% CI: 67.8-86.1), 84.7\% (95\% CI: 74.5-91.0), 95.6\% (95\% CI: 86.9-98.6), and $87.5 \%$ (95\% CI: 77.2-93.3), respectively (Figures 1-4). Four out of 85 (4.7\%) patients experienced a local recurrence. Of these four patients, two had received preoperative radiotherapy. One patient received a preoperative dose of $4400 \mathrm{cGy}$, while the other patient received a preoperative dose of $3000 \mathrm{cGy}$. Out of these, 1 patient $(25 \%)$ had received postoperative radiotherapy. This patient had not received preoperative radiotherapy.

Radiotherapy with or without chemotherapy was associated with a high degree of tumor response. The median percent necrosis for patients who received preoperative radiation therapy was $95 \%$ (range, $0-100 \%$ ), with a mean necrosis rate of $77.6 \%$ (Table 2). Percent necrosis is a direct indicator of pathological response and was inversely associated with histologically intact residual tumor (e.g., $100 \%$ necrosis indicates that no histological tumor was left behind). Ten out of 85 patients had a pathological complete response (11.7\%). On univariate analysis, there was a trend towards higher necrosis rates in patients who received concurrent chemotherapy, $84.7 \%$ (95\% CI: 75.9-93.4) and 69.5\% (95\% CI: 55.1-83.8), $p=0.061$, compared with patients who did not receive concurrent chemotherapy. On multivariate analysis, the use of chemotherapy was significantly associated with increased rates of necrosis (Table 3). However, the addition of chemotherapy did not appear to be associated with improvements in disease-free or overall survival. In patients with tumor size $\geq 5 \mathrm{~cm}$ who had chemotherapy, OS: HR (hazard ratio) is 1.69 (95\% CI: $0.54-5.25), p=0.367$ and DFS: HR is 1.85 (95\% CI: $0.81-4.19), p=0.143$. Tumor size of $<5 \mathrm{~cm}, 5-$ $10 \mathrm{~cm}$, and $>10 \mathrm{~cm}$ has been associated with prognosis in sarcoma subtypes [7].

The use of radiation therapy with or without chemotherapy preoperatively resulted in overall very low rates of local recurrence $(4.7 \%$ in all patients with $2 / 4$ failures occurring in patients with negative margins and $2 / 4$ failures occurring in patients with close $(<1 \mathrm{~mm})$ or positive margins).

With regard to pretreatment variables that could predict outcome, large tumor size, as analyzed by a continuous variable, was associated with inferior disease-free survival and local control. Large tumor size and Grade $\geq 2$ were associated with inferior overall survival (Tables 4-7).

Short- and long-term toxicity was scored based on retrospective chart review and the RTOG acute and late toxicity scale [8]. Acute toxicity data were available for a total of eightythree patients. Seventeen out of 83 patients $(20 \%)$ experienced an RTOG acute skin toxicity Grade 3 or higher. Of these 17 patients, 9 had received chemotherapy (52.9\%), while 8 patients did not receive chemotherapy (47.1\%). These differences were not statistically significant $(p=0.771)$. Late toxicity data were available for a total of 83 patients. Nine patients (10.8\%) experienced an RTOG Grade 3 or higher late skin toxicity.

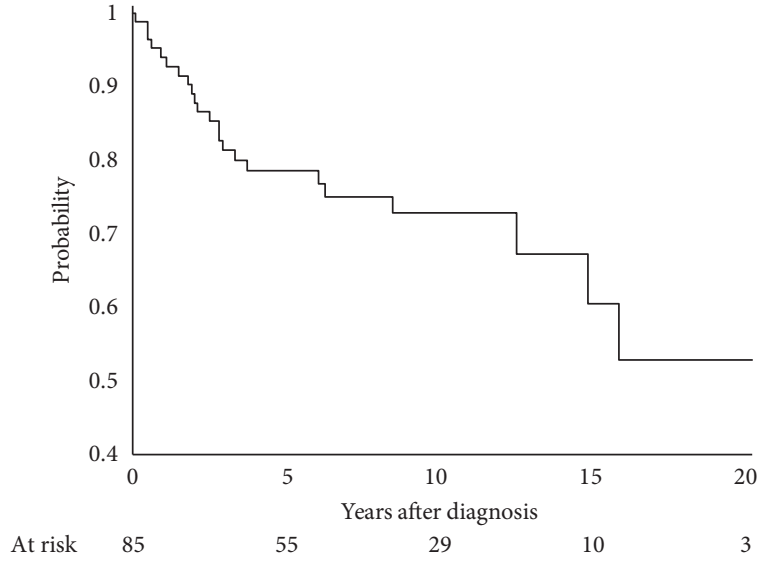

FIgURE 1: Disease-free survival at 5 years, $78.6 \%(95 \% \mathrm{CI}$ : 67.8-86.1).

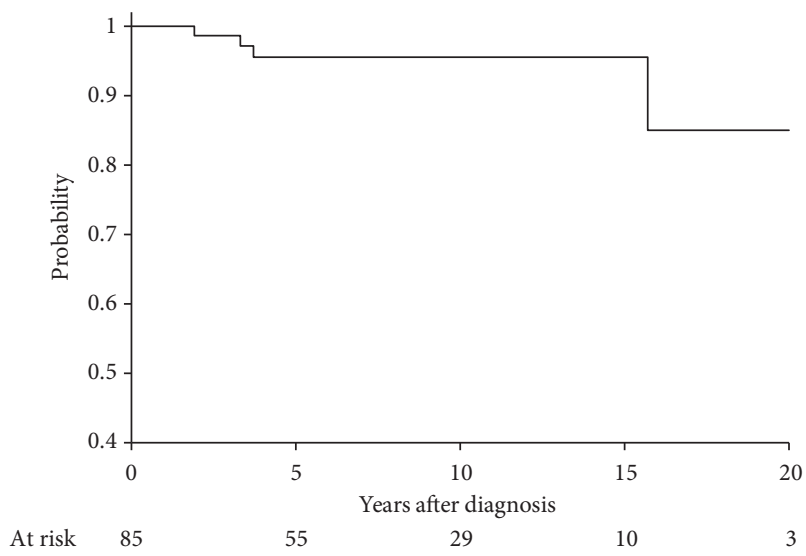

Figure 2: Local control at 5 years, 95.6\% (86.9-98.6).

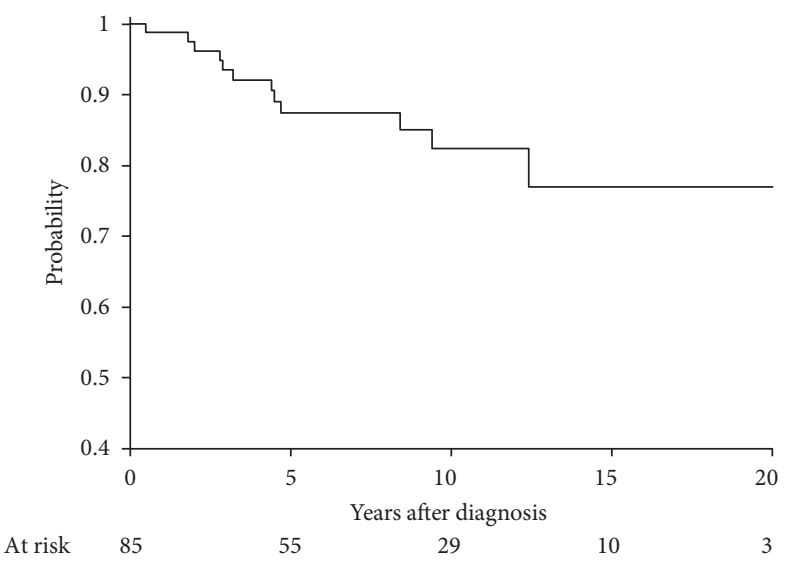

Figure 3: Overall survival at 5 years, 87.5\% (77.2-93.3).

\section{Discussion}

In this series of 85 patients, we found a mean necrosis rate of over $77 \%$ in patients treated with preoperative radiation therapy. These data support the findings from other published series and clinical experience that suggest that MLS is 


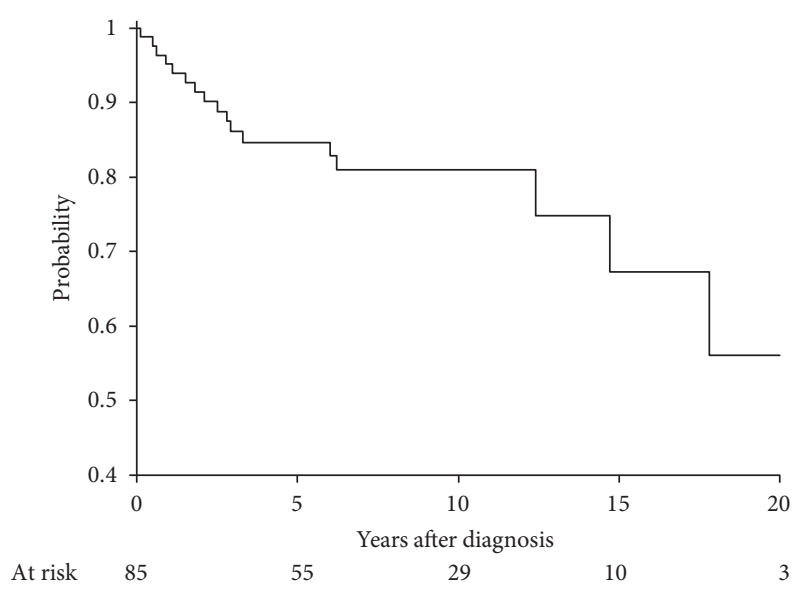

Figure 4: Distant metastases-free survival at 5 years, 84.7 (74.5-91.0).

TABLE 2: Mean tumor response (patients treated with radiation therapy).

\begin{tabular}{lc}
\hline Pre-op RT & $77.6 \%$ \\
No pre-op RT & $0 \%$ \\
Concurrent chemotherapy & $84.7 \%(95 \%$ CI: $75.9-93.4)$ \\
No concurrent chemotherapy & $69.5 \%(95 \%$ CI: $55.1-83.8)$ \\
& $p=0.061$ \\
\hline
\end{tabular}

TABLE 3: Linear regression for tumor response (all patients).

\begin{tabular}{lcc}
\hline Parameter & Linear coefficient \pm SE & $p$ value \\
\hline Use of chemotherapy & $24.6 \pm 9.4$ & 0.011 \\
Margin status & $9.5 \pm 9.3$ & 0.311 \\
Tumor size & $0.9 \pm 0.7$ & 0.209 \\
\hline
\end{tabular}

TABle 4: Local control (all patients).

\begin{tabular}{lcc}
\hline Parameter & Hazard ratio & $p$ value \\
\hline Tumor size & HR 1.17 (per cm) (95\% CI: 1.02-1.33) & 0.022 \\
Margin & HR 0.32 (95\% CI: 0.02-4.49) & 0.396 \\
\hline
\end{tabular}

TABLE 5: Factors associated with disease-free survival on multivariate analysis (patients treated with radiation therapy).

\begin{tabular}{lcc}
\hline Parameter & Hazard ratio & $p$ value \\
\hline $\begin{array}{l}\text { Necrosis/tumor } \\
\text { response }\end{array}$ & 0.90 (per 10\% of necrosis) (95\% CI: & 0.222 \\
$\begin{array}{l}\text { High-grade } \\
\text { disease }\end{array}$ & $5.75-1.07)$ & \\
Tumor size & 1.08 (per cm) (95\% CI: $1.01-1.16)$ & 0.019 \\
\hline
\end{tabular}

a radiosensitive malignancy, as reflected by histological response. While it is not certain how histological response correlates with treatment outcomes, there is a suggestion from some published series that high levels of necrosis may correlate with improved outcomes $[9,10]$. Additionally, the results of our series show a trend that the addition of concurrent chemotherapy increased necrosis to a mean of
TABLE 6: Factors associated with disease-free survival on multivariate analysis.

\begin{tabular}{lcc}
\hline Parameter & Hazard ratio & $p$ value \\
\hline Use of radiation therapy & HR 0.45 (95\% CI: $0.13-1.60)$ & 0.218 \\
Size $>10 \mathrm{~cm}+$ grade $\geq 2$ & HR 3.11 (95\% CI: $1.17-8.27)$ & 0.023 \\
\hline
\end{tabular}

TABLE 7: Factors associated with overall survival multivariate analysis.

\begin{tabular}{lcc}
\hline Parameter & Hazard ratio & $p$ value \\
\hline Any radiation & HR 0.28 (95\% CI: $0.06-1.47)$ & 0.133 \\
Size $>10 \mathrm{~cm}+$ grade $\geq 2$ & HR 6.56 (95\% CI: $1.32-32.76)$ & 0.022 \\
\hline
\end{tabular}

$82.3 \%$ as compared with $58.8 \%$ patients who were not treated with chemotherapy. Our study findings are also consistent with other sarcoma studies in which large tumor size is associated with inferior disease-free survival [11].

The high degree of response supports the use of preoperative therapy of these MLS patients.

While it is our practice to consider additional postoperative boost radiation therapy in patients with positive margins after preoperative radiation, the low rates of local failures make it difficult to perform more detailed analysis of related factors that may impact local control and survival. One of the local failures occurred in a patient who had a grossly positive margin and still had a local failure after postoperative radiation therapy.

The results of our series are concordant with other published series in MLS suggesting chemotherapy sensitivity [12]. Furthermore, our series is hypothesis generating in which chemotherapy may enhance the effect of radiotherapy. In our study, chemoradiotherapy was associated with histological response; we did not see a benefit of chemotherapy with disease-free or overall survival.

While patients in this retrospective series received different chemotherapeutic agents, a large randomized study did not show a benefit to histologically tailored chemotherapy, over a standard regimen [13]. Given the low rate of local failures overall, the number of events make statistical analysis about factors that either enhance locoregional control difficult. From the literature, there is uncertainty whether high necrosis rates correlate with improved outcome. Some reports $[14,15]$ have shown no overall clinical benefit with high tumor necrosis rates, while another has suggested that pathological necrosis does correlate with clinical outcome [16]. Although our series did not demonstrate a definite correlation of the degree of necrosis with disease-free survival or overall survival, it is important to recognize that selection bias likely led to larger, higher risk tumors receiving chemotherapy (which was then associated with greater pathologic necrosis), thus confounding these data. Furthermore, it is possible that mechanisms behind tumor necrosis could vary and that patients with tumor necrosis as a result of a rapidly dividing tumor outgrowing its blood supply could have a different outcome compared with a patient who develops a necrotic tumor due to a significant response. The rarity of this tumor, however, would make a randomized clinical trial designed to address the 
effectiveness of adjuvant chemotherapy specifically for MLS difficult to complete.

The findings of our study support the importance of multidisciplinary care in the management of patients with MLS. In addition to radiotherapy, chemotherapy also appears to enhance the histological response rates to treatment.

Due to the low numbers of local failures, more definitive conclusions on the impact of chemotherapy on local control in MLS cannot be made. The use of concurrent, neoadjuvant chemotherapy, with the potential to reduce the incidence of metastatic disease has a theoretical appeal because of the poor prognosis of patients who ultimately develop metastatic disease [17].

There are some limitations of study that warrant further discussion. First, as a single institution study, there is inherent selection and management bias. The retrospective nature of our series also limits our ability to draw conclusions regarding specific therapies.

Our series did not find any differences in toxicity in patients who received chemotherapy, although it is likely that chemotherapy adds at least some degree of toxicity in patients who are treated with radiation therapy that may not have been captured in a retrospective analysis. It is possible that chemotherapy may have resulted in higher rates of acute Grade 2 or higher skin toxicity; these may not have been captured due to the retrospective nature of our series. Another limitation of this series was that the patients reviewed performed where largely the population of patients was surveilled using CT imaging to detect distant recurrence. There is a recent report that whole-body MRI may be more sensitive at detecting a pulmonary metastases compared with CT, and it is possible that this is more sensitive with regard to the detection of metastatic disease [18]. Furthermore, it is possible that at least some of the patients in this series could have been found to have metastatic disease even prior to the initiation of definitive therapy.

While the addition of chemotherapy provides for a means of treatment intensification in patients with a high risk of local and distant recurrences, the results of our series also present an opportunity to evaluate the role of treatment deintensification. The high rates of local control with a median preoperative radiotherapy dose of 50 Gy suggest that it may be reasonable to de-escalate therapy in selected patients, which is the subject of an ongoing international clinical trial [19]. However, more investigation is required to better understand in which patients such treatment deintensification is safe.

\section{Conclusions}

The results of our study support the notion that myxoid liposarcoma has a high rate of histological response with combined chemotherapy and radiotherapy, with low rates of local failure with trimodality therapy. The high pathological response rates to chemotherapy are hypothesis generating. While some reports [16] have suggested that high necrosis rates may correlate with improved clinical outcomes, our study, while not specifically designed to address this issue, did not demonstrate a correlation between necrosis and clinical outcome. We were not able to show that chemotherapy provides a significant clinical benefit with regard to local control, disease-free survival, or overall survival, although the likely selected usage of chemotherapy in the highest risk patients confounds this analysis. We agree with the National Comprehensive Cancer Network (NCCN) guidelines that chemotherapy should be considered in patients with large, high-grade sarcomas [20]. Further research is required to understand which patients benefit the most from chemotherapy, and which patients may benefit from treatment deintensification.

\section{Data Availability}

The data used to support the findings of this study are available from the corresponding author upon request.

\section{Ethical Approval}

This study was approved by the Institutional Review Board.

\section{Disclosure}

Drs. De Amorim Bernstein and Lozano-Calderón are cosenior authors on this project. This work has been presented as an abstract at the EMSOS 2018, Amsterdam. This work has not been previously published in a peer-reviewed journal.

\section{Conflicts of Interest}

The authors report no conflicts of interest with this work.

\section{References}

[1] C. Antonescu and M. Ladanyi, "Myxoid liposarcoma," in World Health Organization Classification of Tumors, $\mathrm{Pa}$ thology and Genetics of Tumors of Soft Tissue and Bone, C. D. M. Fletcher, K. K. Unni, and F. Mertens, Eds., pp. 40-43, IARC Press, Lyon, France, 2002.

[2] S. E. ten Heuvel, H. J. Hoekstra, R. J. van Ginkel, E. Bastiaannet, and A. J. H. Suurmeijer, "Clinicopathologic prognostic factors in myxoid liposarcoma: a retrospective study of 49 patients with long-term follow-up," Annals of Surgical Oncology, vol. 14, no. 1, pp. 222-229, 2007.

[3] R. S. A. de Vreeze, D. de Jong, R. L. Haas, F. Stewart, and F. van Coevorden, "Effectiveness of radiotherapy in myxoid sarcomas is associated with a dense vascular pattern," International Journal of Radiation Oncology, Biology, Physics, vol. 72, no. 5, pp. 1480-1487, 2008.

[4] G. Pitson, P. Robinson, D. Wilke et al., "Radiation response: an additional unique signature of myxoid liposarcoma," International Journal of Radiation Oncology, Biology, Physics, vol. 60 , no. 2, pp. 522-526, 2004.

[5] R. L. Jones, C. Fisher, O. Al-Muderis, and I. R. Judson, "Differential sensitivity of liposarcoma subtypes to chemotherapy," European Journal of Cancer, vol. 41, no. 18, pp. 2853-2860, 2005.

[6] D. Katz, P. Boonsirikamchai, H. Choi et al., "Efficacy of firstline doxorubicin and ifosfamide in myxoid liposarcoma," Clinical Sarcoma Research, vol. 2, no. 1, p. 2, 2012. 
[7] S. Singer, E. H. Baldini, G. D. Demetri, J. A. Fletcher, and J. M. Corson, "Synovial sarcoma: prognostic significance of tumor size, margin of resection, and mitotic activity for survival," Journal of Clinical Oncology, vol. 14, no. 4, pp. 1201-1208, 1996.

[8] J. D. Cox, J. Stetz, and T. F. Pajak, "Toxicity criteria of the radiation therapy oncology group (RTOG) and the European organization for research and treatment of cancer (EORTC)," International Journal of Radiation Oncology, Biology, Physics, vol. 31, no. 5, pp. 1341-1346, 1995.

[9] P. W. M. Chung, B. M. Deheshi, P. C. Ferguson et al., "Radiosensitivity translates into excellent local control in extremity myxoid liposarcoma: a comparison with other soft tissue sarcomas," Cancer, vol. 115, no. 14, pp. 3254-3261, 2009.

[10] B. A. Guadagnolo, G. K. Zagars, M. T. Ballo et al., "Excellent local control rates and distinctive patterns of failure in myxoid liposarcoma treated with conservation surgery and radiotherapy," International Journal of Radiation Oncology, Biology, Physics, vol. 70, no. 3, pp. 760-765, 2008.

[11] P. W. Pisters, D. H. Leung, J. Woodruff, W. Shi, and M. F. Brennan, "Analysis of prognostic factors in 1,041 patients with localized soft tissue sarcomas of the extremities," Journal of Clinical Oncology, vol. 14, no. 5, pp. 1679-1689, 1996.

[12] S. R. Patel, M. A. Burgess, C. Plager, N. E. Papadopoulos, K. A. Linke, and R. S. Benjamin, "Myxoid liposarcoma. Experience with chemotherapy," Cancer, vol. 74, no. 4, pp. 1265-1269, 1994.

[13] A. Gronchi, S. Ferrari, V. Quagliuolo et al., "Histotypetailored neoadjuvant chemotherapy versus standard chemotherapy in patients with high-risk soft-tissue sarcomas (ISGSTS 1001): an international, open-label, randomised, controlled, phase 3, multicentre trial," The Lancet Oncology, vol. 18, no. 6, pp. 812-822, 2017.

[14] D. R. Lucas, M. P. Kshirsagar, J. S. Biermann et al., "Histologic alterations from neoadjuvant chemotherapy in high-grade extremity soft tissue sarcoma: clinicopathological correlation," The Oncologist, vol. 13, no. 4, pp. 451-458, 2008.

[15] J. T. Mullen, F. J. Hornicek, D. C. Harmon et al., "Prognostic significance of treatment-induced pathologic necrosis in extremity and truncal soft tissue sarcoma after neoadjuvant chemoradiotherapy," Cancer, vol. 120, no. 23, pp. 3676-3682, 2014.

[16] F. C. Eilber, G. Rosen, J. Eckardt et al., "Treatment-induced pathologic necrosis: a predictor of local recurrence and survival in patients receiving neoadjuvant therapy for highgrade extremity soft tissue sarcomas," Journal of Clinical Oncology, vol. 19, no. 13, pp. 3203-3209, 2001.

[17] A. J. Spillane, C. Fisher, and J. M. Thomas, "Myxoid liposarcoma-the frequency and the natural history of nonpulmonary soft tissue metastases," Annals of Surgical Oncology, vol. 6, no. 4, pp. 389-394, 1999.

[18] N. Gorelik, S. M. V. Reddy, R. E. Turcotte et al., "Early detection of metastases using whole-body MRI for initial staging and routine follow-up of myxoid liposarcoma," Skeletal Radiology, vol. 47, no. 3, pp. 369-379, 2018.

[19] The Netherlands Cancer Institute, "Dose reduction of preoperative radiotherapy in myxoid liposarcomas (DOREMY)," 2014, https://clinicaltrials.gov/ct2/show/NCT02106312.

[20] NCCN, "NCCN clinical practice guidelines in oncology. Soft tissue sarcoma. NCCN evidence blocks. Version 2," 2017, https://www.nccn.org/professionals/physician_gls/ pdf/sarcoma_blocks.pdf. 


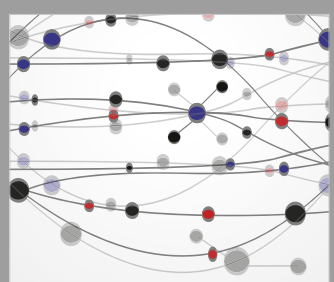

The Scientific World Journal
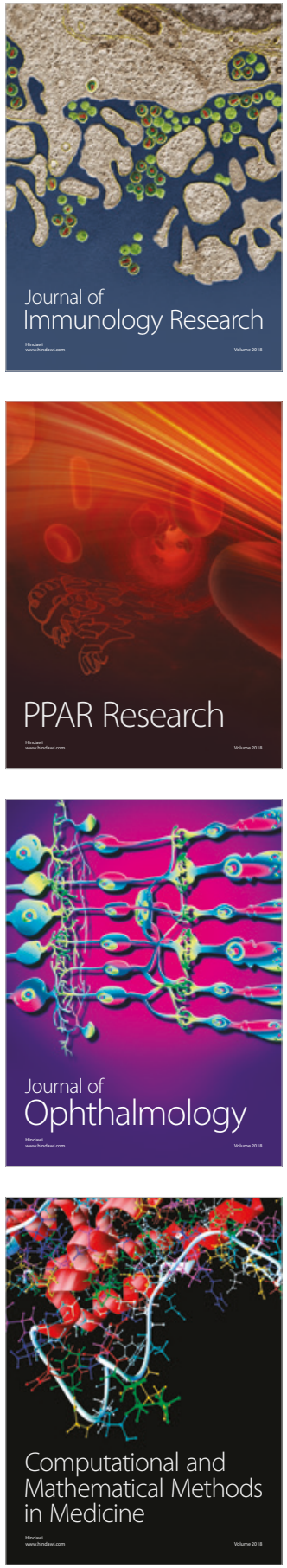

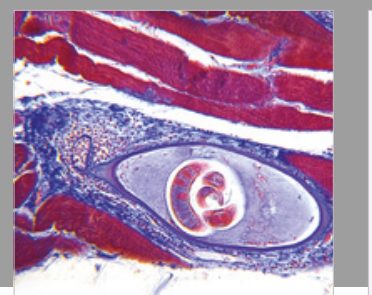

Gastroenterology Research and Practice

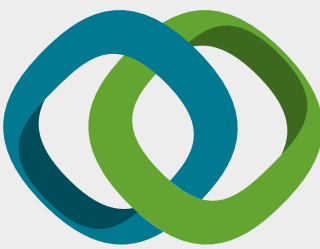

\section{Hindawi}

Submit your manuscripts at

www.hindawi.com
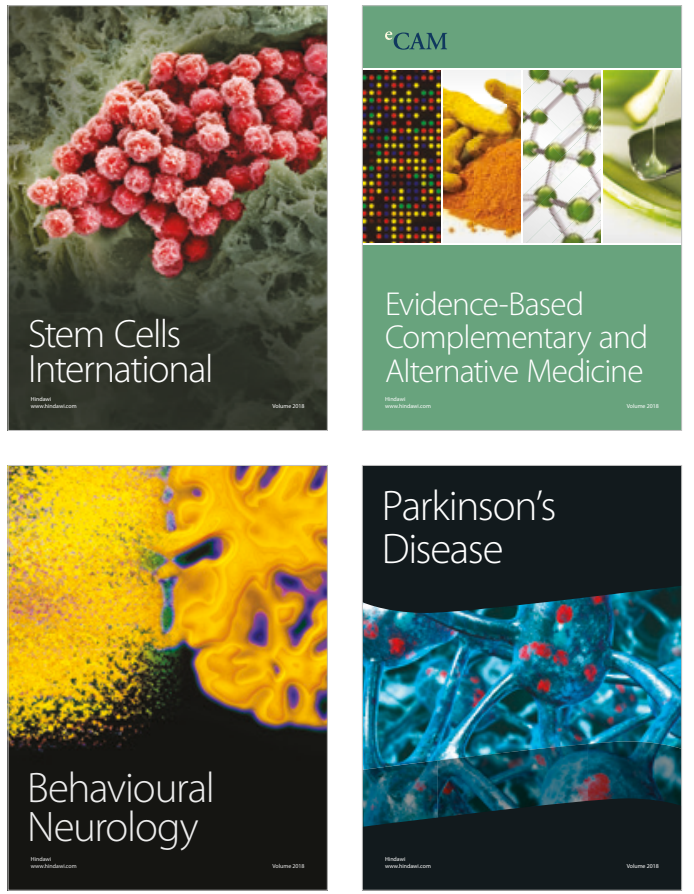

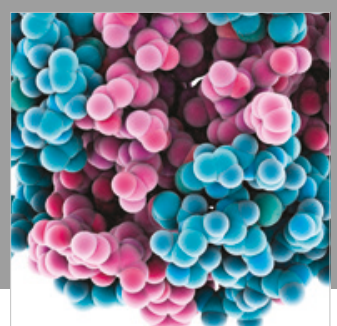

ournal of

Diabetes Research

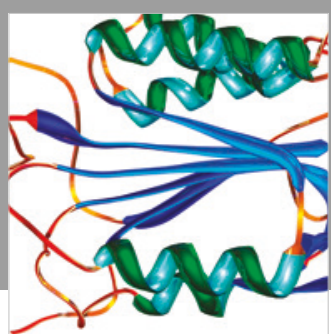

Disease Markers
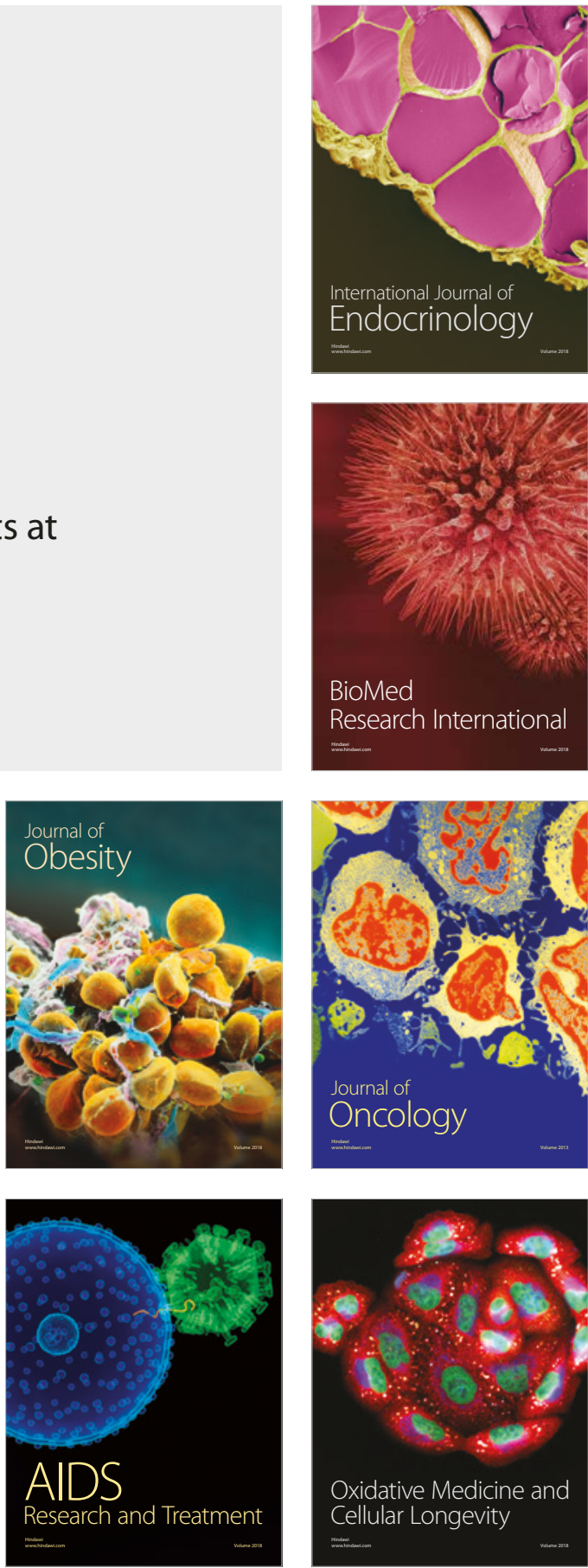